\title{
Un quinquenio de Estudios Generales en el Campus Coto 2010 - 2014
}

\section{Five years of General Studies Program at Coto Campus. Costa Rica. 2010-2014}

\author{
Rodrigo Ignacio Campos Araya \\ Universidad Nacional, Campus Coto \\ ricampos@una.cr \\ Norma Natalia Carrillo Padilla \\ Universidad Nacional, Campus Coto \\ norma.carrillo.padilla@una.cr
}

\section{Resumen}

Los Estudios Generales forman parte esencial de la formación humanística en la Universidad Nacional. Estos han sido parte de un proceso evolutivo en el que se ha dado una apertura evidente en sus distintas áreas. Las Sedes Regionales y distintos campus de la Institución han sido parte de este proceso y han realizado gestiones para que la oferta de estos cursos sea la más atinente, por lo que se presenta un análisis de la situación de estos cursos en el Campus Coto entre el período 2010 - 2014, en cuanto a la oferta de los mismos por áreas, para así determinar algunas conclusiones que permitan ajustar la misma en función del impacto positivo se espera que estas materias tengan en la comunidad estudiantil.

Palabras clave: Estudios generales, Campus Coto, oferta, áreas.

\section{Abstract}

General Studies are an essential part of the humanistic education at the National 
University. These have been part of an evolution process which has produced an evident opening in different areas. The different regional campuses of the institution have been part of this process, and they have made arrangements to strengthen the offer of humanistic courses and to make it more suitable for the professional profiles of the University. This article analyzes the situation of these courses at Coto Campus, from 2010 to 2014, in respect of the offer in different areas. It considers the need for adjusting this offer according to the positive impact in the student community.

Keywords: General Studies, Coto Campus, National University, offer, areas, humanistic education

\section{Introducción}

El Campus Coto de la Universidad Nacional de la Sede Regional Brunca se encuentra ubicado a 5 kilómetros de la frontera con Panamá, en la comunidad de Coloradito, del cantón de Corredores de la provincia de Puntarenas. A la fecha, es uno de los campus más jóvenes, en términos de infraestructura, ya que su edificio fue estrenado en el 2004. Sin embargo, la Universidad Nacional, desde su creación, ha estado presente en la zona, utilizando escuelas y colegios del área en donde se impartían sus programas de educación y otras carreras. De igual forma, desde su creación el Campus Coto ha tenido una marcada dirección hacia el crecimiento, resultado del aumento de la matrícula y de la pertinencia de la oferta académica.

Este trabajo es una investigación exploratoria sobre los estudios generales ofertados en el quinquenio 2010-2014, en el campus Coto de la Universidad Nacional. Se analiza lo que se ha hecho, y se generan conclusiones y recomendaciones en función de lo que a futuro se proyecte en esta importante área del estudio universitario.

El modelo pedagógico de la Universidad Nacional pretende crear un impacto real y necesario en la formación de la población estudiantil que la conforma, para que esta población, una vez que haya terminado su proceso en la universidad, se entregue a la sociedad como profesionales con integridad, ética y humanismo. La mayoría de esta población estudiantil es joven y, según el programa Estado de la Nación (2008), existe un desafío regional consistente en ofrecer trabajo a sus habitantes y eliminar las diferencias sociales; lo anterior nos ubica en la 
realidad espacial a cuya población debemos limitarnos como institución, para que el producto que generemos sea pertinente y pueda, sin ningún problema, ser considerado como un elemento valioso en el sector laboral.

Sergio García (2015) escribe de los supuestos que condicionan la acción educativa "...conocimiento... persona capacitada... sujeto con voluntad". Con base en ellos podemos hablar de que el primero es uno de los principales objetivos de la educación costarricense, donde el aprendizaje y obtención del conocimiento se realiza a través del proceso, que da parte al surgimiento de la persona capacitada que debe convertirse en facilitadora de dicho proceso, para que logre guiar al alumnado en ese camino; finalmente ese alumnado es quien completa esta fórmula, se presenta como aquel sujeto con voluntad de aprender. En función de nuestro modelo se busca que este aprendizaje sea lo más integral posible y despierte siempre la sana crítica y el raciocinio.

Este panorama nos ubica, forzosamente, en la importancia de una rama de los estudios universitarios, definidas como Estudios Generales o Humanidades:

Los Estudios Generales permiten al alumno discernir de manera consiente su vida profesional dejando actuar libremente de manera consciente y madura su elección profesional. Ofrecen al alumno el inicio al que hacer universitario ofreciéndole un espacio académico del saber, proporcionándole una formación humanística e interdisciplinaria. Los Estudios Generales tienen por finalidad, adquirir conocimiento, desarrollar habilidades y valores éticos que formarán parte de su formación que intervendrán en su desarrollo profesional logrando un desarrollo integral (Universidad San Martín de Porres, 2015).

Otra definición de los estudios generales nos permite aportar lo siguiente a la comprensión de estos: "Tronco común de la educación universitaria" que no solo aplicaría para todas las carreras, sino que también cumpliría una función imprescindible en el desarrollo académico de la comunidad estudiantil. Los estudios generales siguen demostrando un aporte necesario, que colaboran en el forje de mejores profesionales, “...una de las actitudes que caracterizan al intelectual crítico es....el pasaporte que se otorga él a sí mismo para moverse a través de y vincular los distintos ámbitos que forman el campo intelectual" (Rojo, 2013, p. 140) y esa vinculación es más fácil de alcanzar a través del estudio de temas relacionados con el arte, las ciencias sociales, las letras, la filosofía, la tecnología, la ética, la historia, entre otras disciplinas. Todas estas humanistas intensifican y amplifican la calidad 
en la formación universitaria, ya que entrelazan intereses, valores, opiniones, análisis y aprecio al conocimiento significativo.

\section{Los Estudios Generales en el Campus Coto}

Durante el período que comprende el estudio (2010-2014) se impartieron en el Campus Coto las carreras de Administración, Ingeniería en Sistemas, Gestión Empresarial del Turismo Sostenible y Enseñanza del Inglés. Como requisito fundamental todo el estudiantado en el sistema educativo universitario público costarricense (independientemente de la facultad), debe aprobar los estudios generales o humanidades. Para el caso de nuestra institución, cada estudiante, por disposición técnica del Centro de Estudios Generales, debe aprobar cuatro cursos, pudiendo llevar dos de la misma área .

La programación de estos cursos, como ya se apuntó, responde a la necesidad que tiene nuestra población estudiantil de estudiar temas universales tendientes a conocer más su región y ampliar la visión del mundo y el aprendizaje significativo. Nos dijo Galileo Galilei que la inercia es la tendencia de los objetos, si están en movimiento, a seguir en movimiento y, si están inmóviles, a permanecer inmóviles (Nájera, 2007). La Universidad debe verse como un ente vivo que, desde su creación, ha estado en una inercia de movimiento, se ha permitido ajustar, replantearse y redirigirse en función de las exigencias que colaboren al logro de los objetivos de crear mejores profesionales.

Algunos estudio, como el de Serna (2010) han expuesto el tema de las humanidades como una necesidad en instituciones de educación general básica, pues según su planteamiento, ayudan a encontrar un verdadero sentido de la vida en sociedad, y este es un tema que no necesariamente inicia en la educación superior universitaria, “...la educación... ejercicio propio de su naturaleza, nació con nosotros, es decir, es parte de nuestra naturaleza y la realizamos en la convivencia social" (Serna, 2010, p. 168). De igual forma nos expone que el ejercicio pedagógico de las humanidades permite discutir, contradecir, pero principalmente argumentar adecuadamente, y justifica lo que decimos, situaciones que vienen a reafirmar la viabilidad y la necesidad generadas de la enseñanza de los estudios generales, y nos hace ver que son temas que deberían incentivarse desde edades más tempranas en los procesos de enseñanza y aprendizaje. 
Nos indica Montiel, que uno de los retos de las humanidades es "la reflexión más contemporánea, porque tengo que aterrizar para llegar hasta nuestros días y hablar de los desafíos que significan en nuestra América" (2010, p.19). Esto nos permite reflexionar en que los estudios generales se convierten también en materias que evolucionan y se ajustan a las exigencias académicas que se enmarcan en las facultades universitarias. No hace mucho tiempo en Costa Rica solo se sabía de cuatro o cinco materias que conformaban el ciclo de humanidades. Actualmente en la Universidad Nacional la oferta de cursos existentes es mayor a 60, lo que manifiesta su evolución y cómo se ha forjado con hechos en la práctica académica de nuestras aulas.

$\mathrm{Al}$ respecto nos aporta el siguiente autor:

En la historia universal una filosofía ha sido original y auténtica no cuando ha planteado simplemente ideas nuevas, sino cuando estas se han correspondido con las exigencias históricas de su momento en los diferentes planos, esto es, en el orden sociopolítico, económico, ideológico y científico. (González, 2007, p. 22)

La temática de la evolución - variedad es defendida por varios autores y autoras en distintos foros a nivel internacional, sobre todo en lo que respecta a las humanidades como respuesta a una formación integral del individuo, y como la capacidad que estos estudios tienen de responder a momentos específicos en la historia, con lo cual generan un aporte al quehacer académico de las unidades en donde se imparten, toda vez que estos siguen un hilo conductor al respeto y aprecio del conocimiento aplicado a la vida cotidiana.

Sin embargo, también es importante mencionar posiciones de autores en relación con la situación que viven los estudios generales "las humanidades se encuentran hoy en peligro de extinción" (Barea, 2014, p. 314); expresión un tanto fuerte y categórica la que nos aporta María Barea donde hace un análisis profundo de la situación en la que, en muchas universidades, se han puestos los estudios generales o humanidades, toda vez que, en un modelo de desarrollo económico y ante una marcada crisis financiera, en muchos países, ante la necesidad de disminuir ciertos gastos, se trata de echar mano de algunos recursos como los dirigidos a la educación superior. De igual forma, nos comenta esta autora que, para la mayoría, estos estudios no marcan una predilección y se categorizan de innecesarios. Ante esta realidad debemos comprometernos para seguir dando el 
espacio que estas materias deben tener en los planes de estudio, y que sigan generando ese amplio espectro académico en estudiantes que las reciben.

Esta investigación se orienta precisamente a observar, analizar, recomendar y concluir en el tanto del alcance que en este quinquenio han tenido los estudios generales en el campus Coto, en apego a una conciencia ética “...la vida humana es sensibilidad, intelecto, memoria, voluntad, es un fin, una multiplicidad de facultades y al mismo tiempo una unidad..." (Berumen, Gomar Ruiz, \& Gómez Danés , 2005, p. 285), expuesta por esta cita de una manera muy interesante, y que nos debe marcar pautas a continuar en el estudio de muchos de los temas referentes a la academia.

\section{Metodología}

Para realizar este trabajo se siguieron los siguientes pasos:

Primeramente, se ingresó al sistema de matrícula de la institución y se consultó la totalidad de la oferta académica, de estudios generales, que se impartieron en los diez ciclos (semestres) correspondientes a los cinco años comprendidos entre el 2010 y el 2014.

Luego de esto se procedió a generar tres instrumentos, uno dirigido a estudiantes de primer ingreso, otro a estudiantes regulares y finalmente uno dirigido a personal académico que imparte cursos generales en el campus.

Luego de la recopilación de datos en el sistema, la aplicación de los cuestionarios a estudiantes y la finalización de entrevistas a docentes, se procedió a tabular la información, analizarla y concluir o recomendar.

La anuencia que encontramos al aplicar y entrevistar fue muy positiva, situación que debe motivar a más temas de investigación en la academia universitaria de aquellas sedes o unidades que se encuentran un tanto alejadas de la sede central, toda vez que es en cada espacio y rincón de nuestra institución donde suceden cosas y acontecen situaciones de suma importancia para los efectos de los objetivos planteados. La realización de este trabajo se debe a que existen insumos que se pueden utilizar en la búsqueda de respuestas que nos permitan visualizar todos aquellos aspectos que debemos mejorar, así como aquellos que hay que reforzar; todo ello con el objeto de que las acciones sustantivas de nuestra institución, como lo son la docencia, la investigación y la extensión, sigan ocupando el mejor de los 
espacios en la agenda institucional y permitan seguir construyendo la universidad necesaria que todos y todas merecemos, y que debemos sostener y fortalecer en función del impacto de esta ante la sociedad civil costarricense.

\section{Resultados del trabajo}

Primeramente, nos ubicaremos en los resultados obtenidos en la recopilación del sistema de matrícula de la Universidad Nacional, en donde se escogieron cinco años que equivalen a diez ciclos lectivos, a razón de dos ciclos por año, entre el 2010 y 2014 (tabla 1).

\begin{tabular}{|c|c|c|c|c|c|c|c|c|c|c|}
\hline \multirow{2}{*}{ Curso } & \multicolumn{2}{|c|}{2010} & \multicolumn{2}{|c|}{2011} & \multicolumn{2}{|c|}{2012} & \multicolumn{2}{|c|}{2013} & \multicolumn{2}{|c|}{2014} \\
\hline & I & II & I & II & I & II & I & II & I & II \\
\hline Introducción a la investigación & $\mathrm{X}$ & -- & $\mathrm{X}$ & -- & $\mathrm{X}$ & -- & $\mathrm{X}$ & -- & $\mathrm{X}$ & -- \\
\hline Ética ambiental & $\mathrm{X}$ & -- & $\mathrm{X}$ & $\mathrm{X}$ & $\mathrm{X}$ & $\mathrm{X}$ & $\mathrm{X}$ & -- & $\mathrm{X}$ & -- \\
\hline Educación, sociedad y ambiente & $\mathrm{X}$ & $\mathrm{X}$ & $\mathrm{X}$ & $\mathrm{X}$ & $\mathrm{X}$ & $\mathrm{X}$ & $\mathrm{X}$ & $\mathrm{X}$ & $\mathrm{X}$ & $\mathrm{X}$ \\
\hline $\begin{array}{l}\text { Terapias alternativas, tradicionales } \\
\text { y complementarias }\end{array}$ & $\mathrm{X}$ & -- & $\mathrm{X}$ & -- & $\mathrm{X}$ & -- & $\mathrm{X}$ & -- & $\mathrm{X}$ & -- \\
\hline Cultura y globalización & $\mathrm{X}$ & -- & $\mathrm{X}$ & -- & $\mathrm{X}$ & -- & $\mathrm{X}$ & $\mathrm{X}$ & $\mathrm{X}$ & -- \\
\hline $\begin{array}{l}\text { Derechos humanos en el nuevo } \\
\text { milenio }\end{array}$ & $\mathrm{X}$ & -- & $\mathrm{X}$ & -- & $\mathrm{X}$ & -- & $\mathrm{X}$ & -- & $\mathrm{X}$ & -- \\
\hline Cultura y adulto mayor & -- & $\mathrm{X}$ & -- & -- & -- & -- & -- & -- & -- & -- \\
\hline Ética y filosofía & $\mathrm{X}$ & $\mathrm{X}$ & $\mathrm{X}$ & $\mathrm{X}$ & $\mathrm{X}$ & $\mathrm{X}$ & $\mathrm{X}$ & $\mathrm{X}$ & -- & $\mathrm{X}$ \\
\hline Teoría y práctica de la creatividad & -- & -- & -- & -- & -- & $\mathrm{X}$ & -- & -- & -- & -- \\
\hline Modernidad y debate ecológico & -- & -- & -- & -- & -- & $X$ & -- & -- & -- & -- \\
\hline $\begin{array}{l}\text { Ciencia, tecnología y sociedad en } \\
\text { el umbral del siglo XXI }\end{array}$ & -- & -- & -- & -- & -- & -- & -- & -- & $\mathrm{X}$ & -- \\
\hline
\end{tabular}

Nota: Sistema de Matrícula de la Universidad Nacional, Sede Regional Brunca, Campus Coto.

La tabla 1 es un resumen de la recopilación de datos del sistema de matrícula, tomó como referencia todos los cursos de estudios generales, impartidos en el campus Coto durante los cinco años comprendidos entre el 2010 y 2014. Es importante aclarar que cada año comprende dos ciclos lectivos (semestres). Esta tabla 1 genera una serie de observaciones que más adelante se procederá a comentar, y que a la postre son las que permitirán crear conclusiones derivadas no solo de esta información, sino también de los instrumentos aplicados a los sujetos participantes y sus opiniones. 
Estos insumos tienen como objeto el poder presentar algunas propuestas devenidas de la investigación, tendientes a apuntar fortalezas o debilidades del proceso de estudios generales en el campus Coto. Podría pensarse en: ¿Qué ha estado bien? ¿Qué debe mejorarse? ¿Cuáles cursos responden mejor a las necesidades del campus? ¿Cuál es la percepción de los sujetos involucrados? Interrogantes que se espera responder con el análisis y desarrollo de la información que se estudia en este trabajo, y que al mismo tiempo deja una puerta abierta a la continuidad investigativa que en función de los resultados se pueda obtener. De igual forma presentamos la figura 2 que muestra, en términos visuales, los cursos de estudios generales y los semestres en que se impartieron en el quinquenio que delimita la investigación.

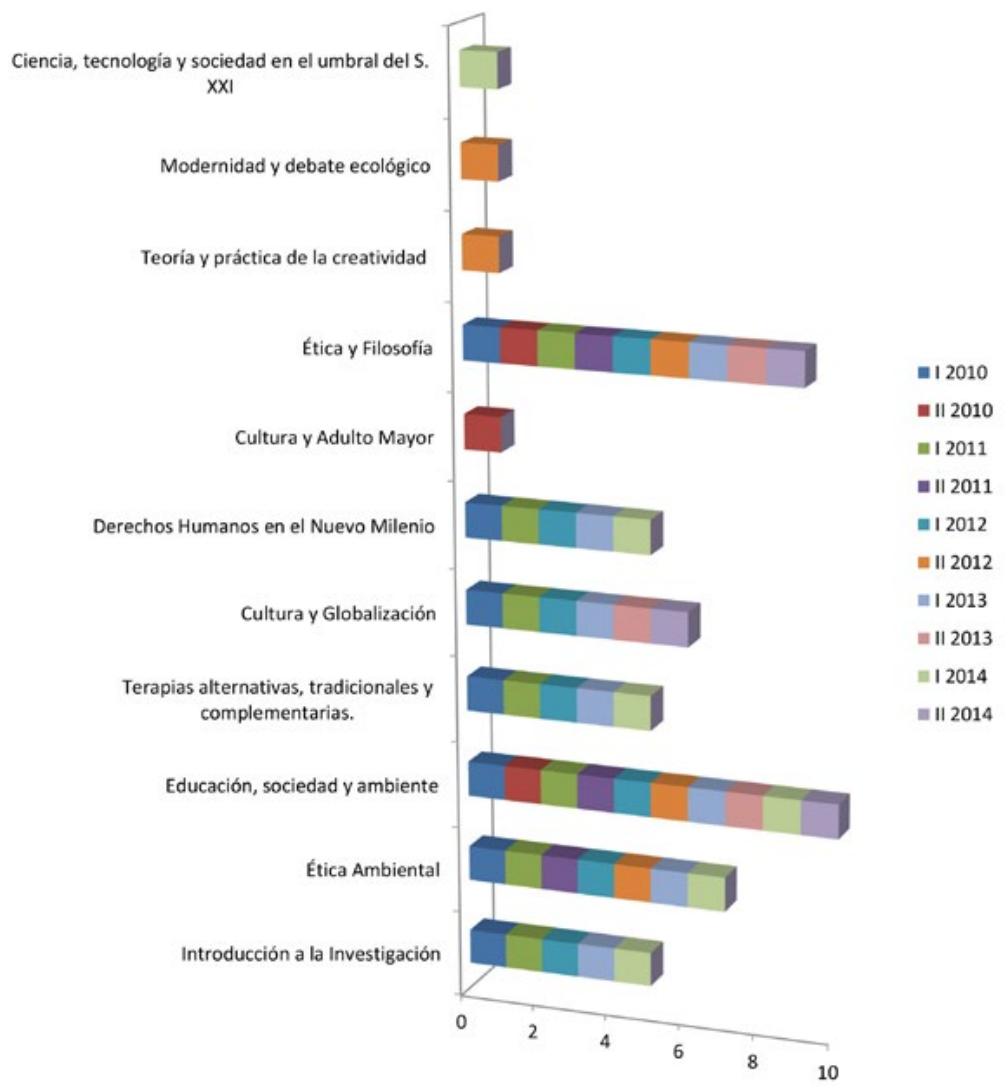

Figura 1: Cursos de Estudios Generales impartidos en el campus Coto 2010 -2014 
Estas son algunas de las observaciones derivadas de las matrículas en estudios generales, ofertas de cursos y demás, acontecidas entre el 2010 y 2014, siendo que la tendencia en la oferta de los cursos, de mayor a menor, se puede describir de la siguiente manera:

En primer lugar, se tiene el curso: Educación, sociedad y ambiente, código EGC108, que fue impartido en el cien por ciento de las ocasiones. El curso: Ética y filosofía, código EGF100, fue ofertado en el noventa por ciento de los períodos. Le sigue el turno a Ética ambiental, código EGC103, que tuvo un porcentaje de 70. En el caso de Cultura y globalización, código EGS101, estuvo en el 60 por ciento de los períodos. Las materias: Introducción a la investigación, código EGA114, Terapias alternativas, tradicionales y complementarias, código EGC109, Derechos humanos en el nuevo milenio, código EGS105, estuvieron presentes en la mitad de los semestres. Finalmente: Cultura y adulto mayor, código EGS107, Teoría y práctica de la creatividad, código EGA102, Modernidad y debate ecológico, código EGC105 y Ciencia, tecnología y sociedad en el umbral del siglo XXI, código EGC100, solo se ofertaron en uno de los ciclos, lo que corresponde a un 10 por ciento.

Esto deja claro que han existido cursos de estudios generales que, por distintas razones, han sido los más tradicionales en la oferta generada para Coto, situación que motiva el objeto de estudio, por tanto uno de los objetivos es valorar la diversificación de los mismos en función de mejoras a la formación integral académica de la comunidad estudiantil, devenidas de las humanidades o estudios generales, sin dejar de lado el hecho de que podrían existir motivos suficientes para que haya cursos que se oferten con más frecuencia. De igual forma se puede afirmar que otros cursos han sido parte importante de la oferta, tal es el caso de aquellos que se encontraron en porcentajes del 70, 60 y 50, donde claramente su presencia fue vinculada al desarrollo de los programas de estudio de las carreras impartidas en el campus. En relación con los cursos de menor porcentaje, es interesante ver que el $36.36 \%$ del total de materias ofertadas en esta categoría solo se dieron en un semestre.

En proporción al número de cursos por área, por semestre, se presenta la tabla 2: 
Tabla 2

Resumen de cursos de Estudios Generales por ciclos y áreas entre 2010 y 2014

\begin{tabular}{lccccccccccc}
\hline \multirow{2}{*}{ Área } & \multicolumn{2}{c}{2010} & \multicolumn{2}{c}{2011} & \multicolumn{2}{c}{2012} & \multicolumn{2}{c}{2013} & \multicolumn{2}{c}{2014} \\
\cline { 2 - 11 } & I & II & I & II & I & II & I & II & I & II \\
\hline Arte / EGA & 1 & -- & 1 & -- & 1 & 1 & 1 & -- & 1 & -- \\
Ciencia y Tecnología / EGC & 3 & 1 & 3 & 2 & 3 & 3 & 3 & 1 & 4 & 1 \\
Filosofía y Letras / EGF & 1 & 1 & 1 & 1 & 1 & 1 & 1 & 1 & -- & 1 \\
Ciencias Sociales / EGS & 2 & 1 & 2 & -- & 2 & -- & 2 & 1 & 1 & 1 \\
\hline
\end{tabular}

Nota: Sistema de Matrícula de la Universidad Nacional, Sede Regional Brunca, Campus Coto.

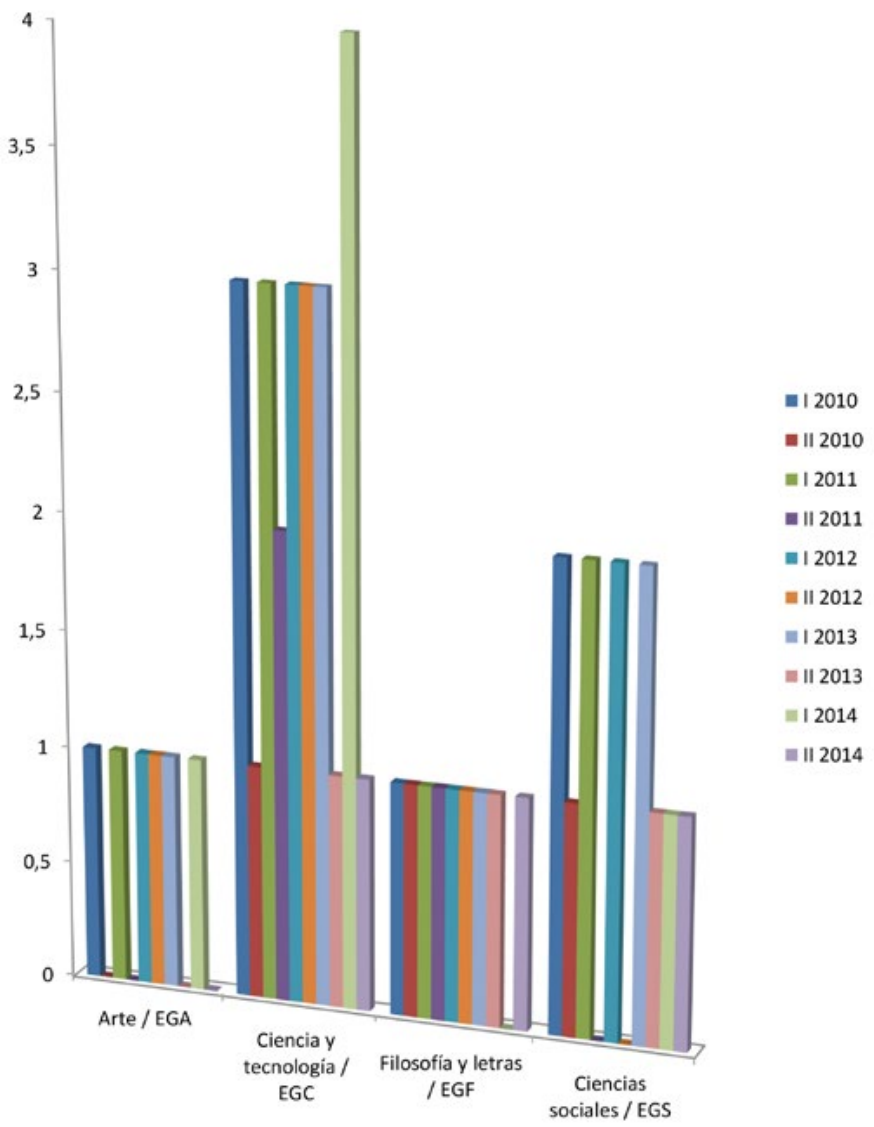

Figura 2: Resumen de cursos por ciclos y áreas entre 2010 y 2014. Elaboración propia basada en tabla 2 
Si nos referimos a las áreas en las que se enmarcan los estudios generales, a saber, arte (EGA), ciencia y tecnología (EGC), filosofía y letras (EGF) y ciencias sociales (EGS), que se exponen en la tabla 2 y figura 2, podemos aportar algunas observaciones.

Primeramente, es importante indicar que en el $40 \%$ de los periodos estudiados fueron ofertadas las cuatro áreas de estudios generales y que, en el $50 \%$ de estos se ofertaron 3 de las 4 existentes. Solamente en uno de los semestres únicamente dos áreas fueron representadas mediante cursos. Únicamente existió un semestre donde esto no se cumplió (II ciclo 2011); pero, para el resto, la variedad en las áreas fue alcanzada. Esta constituye una tendencia rescatable y necesaria, lo cual nos hace ver que en términos de las áreas se ha logrado cumplir con la variedad, requisito de graduación del estudiantado.

A nivel de la totalidad de cursos por área, que en la institución existen, tenemos también el siguiente aporte derivado del periodo de estudio (ver tabla 3):

Tabla 3

Resumen de totalidad, a nivel de oferta institucional, de cursos ofertados en el campus

\begin{tabular}{lc}
\hline \multicolumn{1}{c}{ Área } & Porcentaje \\
\hline Filosofía y letras & 5,00 \\
Arte & 14,28 \\
Ciencias sociales & 17,64 \\
Ciencia y tecnología & 33,33 \\
\hline
\end{tabular}

Nota: Recopilación de datos del Sistema de Matrícula de la Universidad Nacional, Sede Regional Brunca, Campus Coto. 


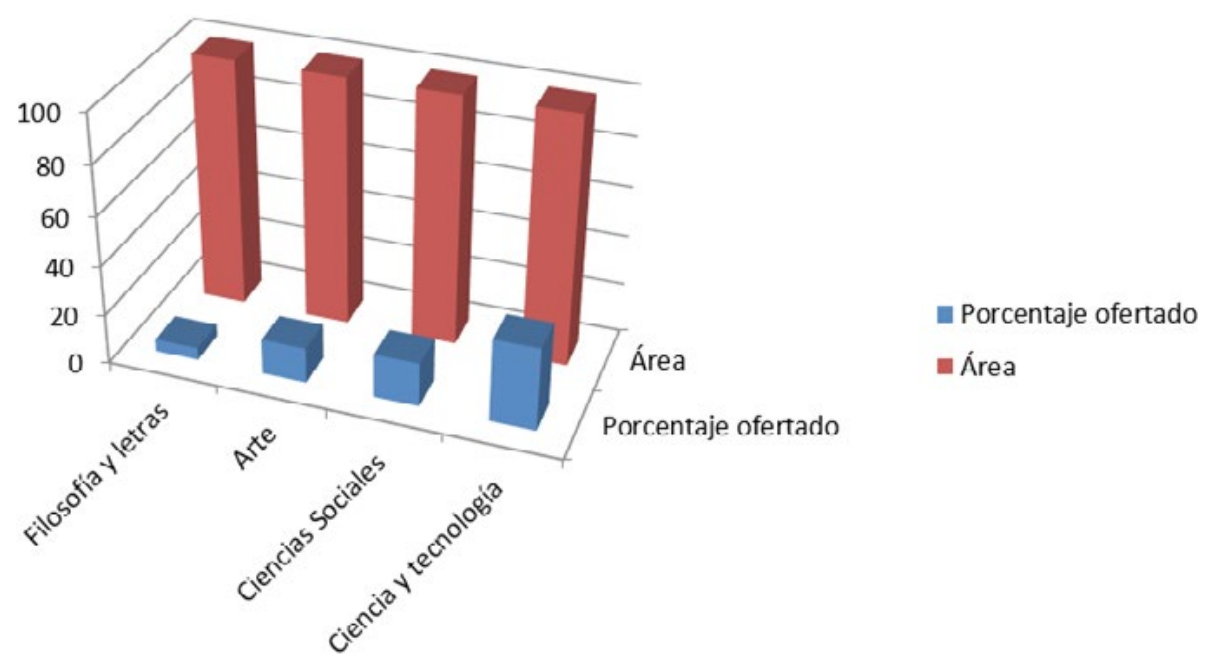

Figura 3: Resumen de totalidad, a nivel de oferta institucional, de cursos ofertados en el campus

Fuente: Elaborado con base en la tabla 3.

Para iniciar a describir lo que esta información aporta, debemos aclarar que tanto la tabla 3 como la figura 3 presentan un análisis de la totalidad de cursos, que a nivel institucional existen, impartidos en el campus Coto, dentro del tiempo estudiado. El caso del menor porcentaje lo tenemos en el área de filosofía y letras, en la que únicamente un $5 \%$ de la totalidad de los cursos existentes se ha podido impartir, le sigue el área de arte en donde el porcentaje de cursos alcanzó un $14,28 \%$, continúa el área de ciencias sociales en donde el porcentaje fue del 17,64\% y finaliza con el área que alcanzó un porcentaje del 33,33\% que es la de ciencia y tecnología.

Es claro que, para un campus pequeño, como lo es Coto, resultaría imposible ofertar la totalidad de cursos generales existentes en la institución, y esto no solo se atribuye al hecho del tamaño sino también a motivos derivados de la matrícula, carreras ofertadas, profesionales en la zona, materiales y hasta infraestructura. En el caso específico se impartieron las carreras de Enseñanza del Inglés, Ingeniería en sistemas de información, Gestión empresarial del turismo sostenible y Administración. 
A continuación, nos ubicaremos en los resultados obtenidos de los instrumentos aplicados a los sujetos que forman la muestra de nuestro estudio, los cuales se determinan en 34 estudiantes regulares, 113 de nuevo ingreso, quienes respondieron un cuestionario. De igual forma, mediante la técnica de la entrevista, obtuvimos los resultados de cinco miembros de la comunidad académica que han impartido cursos generales en el periodo analizado, para lo cual nos permitimos presentar algunos resultados.

El estudiantado regular, al responder las preguntas del cuestionario, generó los siguientes resultados:

- Pregunta1. Como alumno de esta Universidad, ¿tiene usted claridad del concepto de Humanismo o Humanidades? El 23,53\% respondió que sí, mientras que un $11,76 \%$ indicó que no, seguido por un $58,82 \%$ que dijo tener en alguna medida el concepto claro y finalmente el 5,89\% aportó una respuesta en la que indicaron conocerlo en gran medida.

- Pregunta 2. ¿Desde su perspectiva qué tan valiosos fueron los Estudios Generales? El 35,29\% dijo que fueron muy valiosos, mientras que el 29,42\% respondió que eran valiosos, contra un $35,29 \%$ que indicó que eran valiosos en alguna medida.

- Pregunta 3. ¿Cuál fue su curso de Estudios Generales preferido? Esta generó los siguientes resultados: a) primeramente se indica que solo se mencionan 6 de la totalidad de los cursos existentes en la oferta académica de los estudios generales de la Institución; b) los seis cursos mencionados como preferidos incluyen las cuatro áreas de estudio del mismo estudiantado; c) los cursos preferidos son: Introducción a la investigación con un 2,94\%, Derechos humanos en el nuevo milenio con un 5,89\%, Cultura y globalización con un porcentaje de 14,71, Ética ambiental con 17,64\% y finalmente los cursos Ética y filosofía y Educación, sociedad y ambiente, cada uno con un $29,41 \%$. Estos últimos resultaron ser los preferidos y se ubican en el área de filosofía y letras, y ciencia y tecnología, respectivamente.

- Pregunta 4. ¿Considera usted que los profesores que le impartieron cursos de estudios generales en el campus poseen el perfil adecuado para tal efecto? El 52,94\% de las respuestas indican que el profesorado sí poseía el perfil adecuado según el criterio de estudiantes, mientras que el 32,35\% nos aportan una opinión de que lo poseían en alguna medida, finalizando con 
un $17,71 \%$ que opina que los perfiles académicos fueron en gran medida los adecuados para la enseñanza de estos cursos.

En cuanto a la muestra de estudiantes de nuevo ingreso que no han finalizado con los Estudios Generales, se pretendía visualizar su percepción, basada únicamente en su bagaje de la educación secundaria. Esta nos presentó un resultado con las siguientes particularidades: las áreas de estudio, de mayor a menor interés: inicia con ciencia y tecnología, como la más escogida; continúa con ciencias sociales, seguido del área de arte y termina con filosofía y letras, en todas ellas con tendencias a cursos específicos.

Continuando con el análisis de los miembros de la comunidad académica consultada, y utilizando la técnica de la entrevista, presentamos algunas de las opiniones manifestadas en la pregunta: ¿En su criterio, por qué son importantes los estudios generales en las carreras universitarias?

- Académico1: "Me parece que los cursos generales son cursos que brindan el espacio para que el estudiante tenga el cambio del colegio a la universidad. Son cursos donde al estudiante se le da materia de conocimiento general. Deben existir en el primer año de carrera."

- Académico 2: "Permiten al estudiante tener una visión más amplia de la vida, generar discusiones y análisis con temas diversos de la sociedad actual, ampliar conocimientos en diversas áreas."

- Académico 3: "Son cursos que favorecen el entorno universitario, ayudan a la elección vocacional, además de una adecuada preparación para el inicio profesional. Desarrollan aspectos como la ciencia y la cultura en su propia existencia. Fortaleciendo el enfoque humanista y su cultura."

- Académico 4: "Aporta una visión de mundo que "humaniza" al profesional."

- Académica 5: "Porque ubica y ponen en contexto universal al estudiante. Le dice en dónde está él o ella en el mundo; y cómo está ubicada su carrera en el mismo. Además son cursos que los harán mejores estudiantes en su carrera, ya que les proporcionan instrumentos teóricos y de información referencial."

En relación con los aportes indicados por los académicos y académicas entrevistadas procedemos a citar algunos: "Una visión crítica de la realidad, el análisis de diversos pensamientos presentes en la sociedad. Considero que soy un mediador para que los estudiantes analicen y establezcan sus propios 
criterios. Un facilitador, del conocimiento en diversas áreas. El aprendizaje significativo"; "Motivar al estudiante a establecer una opinión más amplia, social y comprometida"; "Un enfoque humanista, conocimiento, interacción entre estudiantes y docentes, medio ambiente, valores, letras, etc."; "He tratado de aportar lo que desarrollé en el punto o respuesta número 1, además siempre incluyo aspectos de ética profesional y los ejes transversales de la UNA, a saber, género, ambiente, equidad etc."

De esta forma se concluye con el análisis de los datos y resultados obtenidos en la investigación.

\section{Conclusiones y recomendaciones}

Iniciamos esta sección con la siguiente cita “...el bien propio del hombre no radica en lo que éste se imagina o desee a capricho, sino en lo que él es, y esto indico sólo un determinado rango de posibilidades" (Berumen, Gomar Ruiz, \& Gómez Danés , 2005, p. 378). Esta se desliga de un análisis de la ética en el ejercicio de la función profesional y, de igual forma, aporta una serie de distintos matices que nos hacen reflexionar sobre la seriedad con la que se debe tomar el diseño y programación de las mallas curriculares que definen las carreras de nuestra universidad. Proponemos que sigan siendo reflejo real de la presencia de valores humanistas y que proporcionen espacios de desarrollo académico en las áreas de los estudios generales, pues estos son los que complementan la formación integral de profesionales, aportando criterios éticos basados en la realidad en la que vivimos y en las posibilidades que se tienen para mejorar dicha realidad; se genera, así, una mejor visión de la cultura. "Nietzsche considera que el Estado, la economía y la política deben tener como centro fundamental la cultura" (Vilchis Peñalosa, 2010, p. 106). A manera de comparación, podríamos aportar también el hecho de que la cultura de los estudios generales debe ser el centro fundamental en la academia universitaria en pro de la calidad de esta.

A pesar de que la programación en estos cinco años ha tenido una variedad que incluye las cuatro áreas de estudios, es evidente la tendencia que mostró el alumnado de nuevo ingreso ante los cursos que le gustaría llevar, esto deja a las áreas de arte y filosofías y letras como las áreas menos estudiadas en el campus Coto y, por tendencia, las que menos se estudiarán. Ante esta situación se recomienda a las autoridades académicas tomar en cuenta las preferencias 
mostradas por la comunidad académica, sin dejar de lado la importancia de que todas las áreas estén presentes en la formación académica del estudiantado.

El área que más cursos ofertados tuvo, ciencia y tecnología, apenas ha logrado un $33,33 \%$ de la totalidad de cursos que en esa ella existe, en relación con las otras tuvimos resultados inferiores al $20 \%$ y en un caso fue del $5 \%$, lo que ha dejado de manifiesto que existe una amplia cantidad de cursos que nunca se han ofertado en el campus, donde los resultados hablan por sí solos. Se propone un análisis de todos los datos recopilados, así como las opiniones y preferencias manifestadas, para revisar la posibilidad de diversificar la oferta académica de estos cursos; esto, en apego estricto a las posibilidades que en la zona existan de profesionales en las distintas áreas y, por supuesto, de la atinencia de estos cursos en las carreras estudiadas.

Es interesante ver cómo en el área de filosofía y letras existe un 95\% de cursos que nunca se han impartido en el campus. Hay muchas razones que podrían explicar esa situación, sin embargo, consideramos importante un análisis que determine la pertinencia de los cursos de esta área, de manera que puedan ofrecerse en un mayor número en el campus, ya que esto podría generar también un cambio en la percepción que presenta la comunidad estudiantil universitaria respecto de esta área.

A través de este trabajo se han analizado las realidades de nuestro campus, las cuales han afirmado el impacto que los estudios generales tienen en el alumnado en relación con lo que estos aportan. Es importante seguir validando la presencia de estos estudios, de manera que nuestra población estudiantil adquiera certeza de la gran importancia de ellos en el desarrollo integral de los futuros grupos profesionales y en una superior capacitación de ellos para insertarse en el mercado laboral.

Consideramos también que los estudios generales deben generar un impacto más allá de la Institución, de manera que en estas materias también se materialicen ejemplos de la extensión universitaria, y que esta misma sea una iniciativa compartida por academia y estudiantes.

Finalmente, es importante mencionar la jerarquía que estos temas tienen en el ámbito de la investigación académica, ya que aportan una visión clara y concreta de las realidades que se viven en las distintas sedes de la Universidad, por lo tanto, estamos convencidos de que, en los próximos cinco años, se tendrá que 
hacer otro análisis concatenado con los resultados que obtuvimos en este, para seguir de cerca el desarrollo y percepción de impacto que estos cursos generan. Por ahora, nos quedamos con la idea y esperanza de que en el campus Coto sigamos investigando y analizando los temas que competen a realidades, instituciones nacionales e internacionales, de manera que podamos fortalecer cada día más nuestra acción sustantiva y podamos reafirmar más la necesidad que representamos para la sociedad, sobre todo en comunidades en donde los índices de desarrollo humano marcan las mayores carencias en el país.

\section{Referencias}

Molina B., M. C., (2014) Sobre la necesidad de las humanidades en la formación académica. Revista de Fomento Social, no 275 (Julio-septiembre), 315-339.

Berumen, N. D., Gomar Ruiz, S., \& Gómez Danés, P. (2005). Ética del ejercicio profesional. México: Continental.

Osorio, S.N. (2015). Formación integral en el ámbito universitario: Acerca de la "enseñanza" de las humanidades en la educación superior. Revista Educación y Desarrollo Social. 9(1), 28-39.

González, P. G. (2007). Humanismo en el pensamiento latinoamericano del Siglo XXI. Memoria Congreso Internacional de Humanidades. Heredia, Costa Rica: Centro de Estudios Generales.

Montiel, E. (2010). Los retos de las humanidades ante la realidad latinoamericana. Revista Cultura de Guatemala, Jornadas Humanísticas Landivarianas, El rol de las humanidades en el siglo XXI. Año XXXI Volumen III septiembre diciembre 2010.

Nájera, J. M. (2007). El ser humano en su entorno. San José: EUNED.

Programa Estado de la Nación, (2008). Decimocuarto Informe Estado de la Nación en Desarrollo Humano Sostenible. San José, Costa Rica: Programa Estado de la Nación.

Rojo, G. (2013). Estropicios académicos y tanteos críticos. Revista Chilena de Literatura, 0 (84). Recuperado de http://www.revistaliteratura.uchile. 
cl/index.php/RCL/article/view/28508/39120 Doi: 10.4067/S071822952013000200009

Serna S., P. P., (2010). De la enseñanza de las humanidades en la educación básica. Zona Próxima, () 166-173. Recuperado de http://www.redalyc.org/ articulo.oa? $\mathrm{id}=85317326012$

Universidad San Martín de Porres. (2015). Estudios Generales. Recuperado de http://www.usmp.edu.pe/estudiosgenerales/

Vilchis Peñalosa, J. (2010). Industria de la cultura e influencia en la formación del carácter narcisista.En Hernández Baqueiro, A. Ética actual y profesional, lecturas para la convivencia global en el siglo XXI. México: CENGAGE Learning. 